\title{
CIENCIAS DEL COMPORTAMIENTO Y POLÍTICA: TIEMPO DE “EMPUJAR” LA CONDUCTA DE LOS GOBERNANTES ${ }^{\circ}$
}

\author{
BEHAVIORAL SCIENCES AND POLITICS: TIME \\ TO NUDGE PUBLIC OFFICIALS \\ Sebastián Linares* \\ Esteban Freidin*
}

enviado:14 marzo 2017 - aceptado: 24 marzo 2017

\begin{abstract}
Resumen
El enfoque de Behavioral Insights (BI) implica utilizar conocimientos de las ciencias del comportamiento en el diseño, implementación y evaluación de políticas públicas. En general, los proyectos asociados a BI buscan afectar la conducta de los ciudadanos en sentidos socialmente deseables. En este artículo planteamos que el mismo enfoque puede ser utilizado como marco para "pensar" cómo moldear la conducta de los gobernantes con fines prosociales. Con este objetivo, discutimos dos conceptos: 1) la alineación de los incentivos de gobernantes y ciudadanos, y 2) el enfoque Nudge, que implica estructurar los ambientes de decisión para guiar a los agentes hacia fines prosociales. Concluimos mencionando algunas promesas y limitaciones del enfoque BI en general y de nuestras propuestas específicas en particular.
\end{abstract}

Código JEL: D03, D04, D72, H19

Palabras claves: psicología económica, economía conductual, gobernantes, responsabilidad del piloto, alineación de incentivos.

\footnotetext{
* Instituto de Investigaciones Económicas y Sociales del Sur (IIESS) CONICET Bahía Blanca Correos electrónicos: slinares@iiess-conicet.gob.ar, efreidin@iiess-conicet.gob.ar

`Linares S. \& Freidin, E. (2017). Ciencias del comportamiento y política: tiempo de "empujar” la conducta de los gobernantes. Estudios económicos, 34 (69), 71-87.
} 


\begin{abstract}
The Behavioral Insights (BI) approach involves the use of knowledge from the behavioral sciences in the design, implementation, and evaluation of public policy. In general, projects associated to BI attempt to affect citizens' behavior in socially desirable ways. In this article, we propose that the same approach can be used as a framework to "think" how to shape government officials" behavior with prosocial ends. With this goal in mind, we discuss two concepts: 1) the alignment of incentives between government officials and citizens; and 2) the Nudge approach, which implies structuring decision environments to guide agents towards pro-social ends. We conclude by mentioning some promises and limitations of the BI approach, generally, and of our specific proposals.
\end{abstract}

JEL Code: D03, D04, D72, H19

Keywords: economic psychology, behavioral economics, government officials, pilot's responsibility, alignment of incentives.

For every bias identified for individuals, there is an accompanying bias in the public sphere.

Cass R. Sunstein ${ }^{1}$

\title{
INTRODUCCIÓN
}

\section{ENFOQUE DE BEHAVIORAL INSIGHTS (BI)}

El enfoque BI se basa en los principios y generalizaciones derivados de estudios científicos del comportamiento y la toma de decisiones. Se trata de herramientas, tanto de contenido como metodológicas, provenientes del campo de las ciencias del comportamiento (e.g., economía del comportamiento, psicología social, neurociencias, etc.) que pueden utilizarse para intervenir en los distintos

1 Sunstein, C. R. (2012). Why Nudge? The Politics of Libertarian Paternalism. New Haven: Yale University Press. 
momentos del ciclo de vida de las políticas públicas (Sousa Lourenço, Ciriolo, Rafael Almeida y Troussard, 2016).

Según el reporte 2016 de la Unión Europea (Sousa Lourenço et al., 2016), el enfoque BI puede contribuir en la elaboración, implementación y ajuste de políticas públicas, al menos, de tres maneras distintas: 1) las iniciativas probadas refieren a propuestas basadas en pruebas empíricas ad hoc o experimentos adecuadamente escalados luego de un estudio inicial; 2) las iniciativas informadas refieren a propuestas cuyo diseño se basa en evidencia científica ya existente; y 3) las iniciativas alineadas refieren a propuestas que, al menos a posteriori, son consistentes con la evidencia científica disponible. La fuerza general de este abordaje radica en que se apoya en evidencia empírica y metodología científica para contribuir al diseño y evaluación de políticas públicas. De esta manera, contribuye a generar supuestos realistas sobre la conducta de las personas sobre los que se pueden diseñar políticas mejor enfocadas y más efectivas (Sousa Lourenço et al., 2016).

La transparencia en los distintos momentos implicados en el desarrollo, prueba e implementación de políticas públicas es otra de las virtudes del enfoque BI. En la fase de desarrollo, permite explicitar los principios y resultados experimentales que son tenidos en cuenta al momento de elaborar las distintas regulaciones, normas y políticas en discusión. En la fase de prueba, promueve la utilización de métodos estandarizados que permiten realizar inferencias sólidas a partir de los datos recabados. La misma metodología científica pone énfasis en procesos apropiados de recolección y análisis de los datos. Lo mismo aplica a etapas de seguimiento. Por último, la justificación acerca de la implementación de una política y no otra presenta un fundamento explícito en el proceso realizado y los resultados obtenidos. Cada una de estas etapas puede ser adecuadamente informada al público, lo que probablemente genere confianza en el gobierno, legitimidad en las regulaciones implementadas, así como una adecuada comprensión de los diversos procesos utilizados en el ciclo de vida de las regulaciones y políticas.

En los últimos años, varios organismos internacionales como el Banco Mundial y la Comisión Europea han reconocido el aporte valioso que representa el enfoque BI (Sousa Lourenço et al., 2016). La Unión Europea se ubica como uno de los gobiernos pioneros en implementar y desarrollar el enfoque BI, principalmente a través de la organización de equipos de especialistas en los gobiernos de varios países miembros. Reino Unido se presenta como una de las cabezas en la implementación del enfoque BI y así ha generado y puesto a prueba una variedad de políticas en dominios tan diversos como el transporte, la salud pública, la defensa al consumidor y el cuidado del medioambiente (The Behavioral Insights 
Team, 2016). Recientemente, Barack Obama también convocó a dar prioridad al desarrollo de este enfoque en el gobierno de EE. UU. (Obama, 2015). En la Argentina, hay un equipo del gobierno de la Ciudad de Buenos Aires que, afortunadamente, también está buscando maneras de nutrirse del enfoque BI (Ramiro Álvarez Ugarte, comunicación personal). En este sentido, el enfoque BI se presenta como una visión innovadora y complementaria a las formas tradicionales de gobierno que promete transparentar el proceso de elaboración, implementación, seguimiento y evaluación de normativas, así como dotarlo de mayor asidero científico y empírico. El resultado es una forma de gobierno más responsable y que adquiere mayor legitimidad.

Como puede observarse, por ejemplo, en los reportes de la Unión Europea (Sousa Lourenço et al., 2016) y de Reino Unido (Behavioral Insights Teams, 2016), el enfoque BI ha tenido como objetivo preponderante lograr cambios socialmente deseables en el comportamiento de los ciudadanos. Los ejemplos van desde promover comportamientos proambientales, como lograr un uso responsable de la energía o la adecuada organización de los residuos domésticos para facilitar el reciclado de materiales reusables, hasta estimular a los contribuyentes morosos a ponerse al día con sus cuentas, pasando por buscar maneras de incrementar la donación de órganos. Estas aproximaciones han resultado efectivas en la modulación de la conducta de las personas en direcciones socialmente deseables.

Nuestra principal propuesta en este trabajo es pensar cómo aplicar el enfoque BI para abordar cambios socialmente deseables, no en el comportamiento de los ciudadanos, sino en el comportamiento de los gobernantes. A continuación, introducimos dos nociones que son relevantes para pensar en la aplicación del enfoque BI en este último sentido. Primero, discutimos la importancia de alinear los incentivos de los gobernantes con los de los ciudadanos (que denominaremos la “responsabilidad del piloto”; López Guerra, 2016). Esta idea adquiere relevancia cuando se da de baja el supuesto irreal e ingenuo de que los gobernantes necesariamente eligen aquello que es mejor para los gobernados, en lugar de verse sesgados por sus propios intereses y prácticas plagadas de nepotismo. En segunda instancia, planteamos la utilización de la estrategia nudge (Thaler y Sunstein, 2008), que busca estructurar los ambientes de decisión para facilitar los comportamientos socialmente deseables. 


\section{LA RESPONSABILIDAD DEL PILOTO}

Con frecuencia se ha planteado el símil entre la relación de los ciudadanos con el gobierno de una sociedad y la relación de los pasajeros con el piloto de un avión: así como ponemos nuestra confianza en las competencias del piloto del avión para llegar a buen puerto, también tenemos la obligación de confiar en los sabios o expertos para la conducción de los negocios públicos². El símil ha servido, durante mucho tiempo, para justificar la aristocracia o el gobierno de los autoseleccionados mejores (no electos), pero más tarde también para justificar la democracia estrictamente electoral, esto es, las elecciones populares de los “mejores” y el monopolio del control de la agenda en sus manos.

Se supone que las elecciones competitivas tienen como cometido la "alineación” entre las preferencias de la mayoría de los ciudadanos y las políticas públicas resultantes. Dado que los gobernados escogerán a los gobernantes, los que buscan acceder al poder en teoría ofrecerán políticas ajustadas a los intereses y preferencias de los gobernados. Los pasajeros del avión confían en que el piloto conducirá el avión a buen puerto porque asumen que a) tiene adecuados conocimientos de navegación área y b) si comete algún error, él también sufrirá las consecuencias negativas. De manera análoga, los ciudadanos de una sociedad democrática deberían confiar en sus representantes por razones similares. Primero, porque se supone que son los mejores para gobernar, a menos a los ojos de la (mayoría de la) ciudadanía ${ }^{3}$. Y segundo, porque se supone - ahora veremos que no necesariamente es así- que los incentivos de los gobernantes al gobernar están alineados con el mejor interés de los ciudadanos: si hacen un mal gobierno, entonces sufrirían en carne propia las malas políticas y los gobernados los echarán del poder.

La elección popular de representantes, sin embargo, conlleva dos grandes peligros: el peligro de que los gobernantes incumplan las promesas hechas a la mayoría y gobiernen en beneficio de los grupos más poderosos, y el peligro de que los representantes se aprovechen de su posición de poder para extraer privilegios, rentas extraordinarias y ventajas económicas en beneficio propio y de sus allegados. El primer riesgo es el de la "legislación de clase”. El segundo peligro

2 La palabra en griego para designar a quien dirige el barco es kubernetes, que en latín se tradujo como gubernare, 'gobernar'. Así pues, la idea de la navegación queda muy próxima a la de dirigir una ciudad, una polis.

3 Así veía las elecciones Madison, uno de los fundadores del gobierno representativo moderno: “un gobierno de ciudadanos elegidos, cuya sabiduría pueda discernir mejor los verdaderos intereses de su país, y con un patriotismo y amor por la justicia muy poco probable de ser sacrificado en favor de consideraciones temporales o parciales” (Federalist, 10, para. 16). 
se manifiesta en dos conductas indeseables: a) la corrupción (como cuando un funcionario acepta sobornos) y b) el conflicto de interés en las decisiones (como cuando un funcionario se aprovecha de información pública privilegiada para realizar inversiones privadas que lo enriquecen). Los dos peligros comportan un problema para la teoría democrática porque distorsionan y contaminan el proceso de comunicación y agregación de preferencias, torciendo el sistema hacia el lado de una mayor desigualdad económica y una mayor concentración de la riqueza en pocas manos. Es verdad que en la medida en que esta forma de gobernar sea explícita (por ende, conocida por los ciudadanos), los gobernantes probablemente pagarían las consecuencias en las urnas (en la siguiente votación). No obstante, la corrupción justamente suele desarrollarse en gran parte de forma oculta, lo cual dificulta que se logre responsabilizar a los gobernantes que sacan provecho personal de su puesto público. De hecho, las evidencias muestran de manera casi unánime que el precio electoral de la corrupción es muy bajo 4 .

Es muy posible que, en general, el funcionamiento de las democracias electorales no se acerque tanto a la metáfora del avión piloteado por un virtuoso capitán. Los gobernantes, como el resto de los ciudadanos, son personas que además de sus motivaciones prosociales y altruistas, cuentan con sesgos que, de manera estratégica o no, los pueden inclinar en direcciones egoístas o favorables al nepotismo (i. e., favorecer a familiares, amigos o compañeros de negocios). En la ciencia política contemporánea existen diversas propuestas para reducir estos peligros. Una vertiente de estas propuestas apunta a la influencia de los grupos de interés en las campañas electorales, y busca neutralizar tanto como se pueda la perniciosa injerencia del dinero en la política. Una segunda vertiente busca regenerar el sistema de "pesos y contrapesos" o controles horizontales del poder, que fue concebido precisamente para introducir sanciones efectivas contra los representantes en casos de corrupción. Una tercera vertiente, que es el enfoque que nosotros queremos introducir como insumo para la discusión de una agenda de transparencia e innovación institucional, abreva de las ciencias del comportamiento y se propone

4 Esto es lo que se ha dado en llamar la "paradoja de la corrupción”. Es decir, los políticos, aunque realicen actividades corruptas, resultan populares y sobreviven a las elecciones. Los datos confirman esta teoría. Se observa, en esa línea, que el 70 \% de los alcaldes acusados de corrupción han sido reelegidos, aunque algunos investigadores encuentran que existe un decrecimiento entre el 9,7 \% y 11 \% de su apoyo (Costas-Pérez, Solé-Ollé y Sorribas-Navarro, 2012). También Rivero-Rodríguez y Fernández-Vázquez (2011) analizan los casos de corrupción municipal en España en los que alguna autoridad judicial ha intervenido (para considerar la corrupción más creíble), entre 1999 y 2007. La conclusión a la que llegan estos autores es que los candidatos corruptos, que se mantienen en su partido, obtienen de media los mismos que los no corruptos. 
reflexionar sobre la alineación de incentivos adecuada para que el gobernante (piloto) cumpla sus promesas y ejerza sus funciones con responsabilidad.

Según este tercer enfoque normativo y conductualmente informado, debemos articular un sistema que asegure que el gobernante — como el piloto- se vea afectado "personalmente" por las consecuencias de las decisiones colectivas que toma (López Guerra, 2016). Así como tenemos la certeza de que un piloto de avión tiene incentivos para ser responsable porque cualquier error grave en la dirección del vuelo pondría en peligro su propia vida, debemos articular las instituciones de tal manera que "el piloto de la sociedad" experimente la misma suerte que la ciudadanía. Se trata, por tanto, de pensar de manera inteligente en cómo alinear los incentivos que enfrentan funcionarios y ciudadanos, de tal manera que los primeros, aun estando sesgados de manera egoísta en su toma de decisiones públicas, se vean inclinados en direcciones que favorezcan a la ciudadanía también.

Una situación que serviría para ejemplificar la noción de la responsabilidad del piloto y la alineación de incentivos entre gobernantes y gobernados podría vincularse a la calidad de la educación pública y el funcionamiento de las instituciones asociadas. En una sociedad en la que la calidad educativa en las instituciones públicas es deficitaria, puede ocurrir que ciertos sectores de la población (las clases más pudientes, en las que se encuentran los gobernantes) opten por enviar a sus hijos a escuelas privadas que prometan una mejor educación. Es menos que ideal que los gobernantes y funcionarios públicos encargados de lograr mejoras en el sistema educativo no se vean directamente afectados por la calidad de este sistema. Nuestra propuesta de alineación de incentivos justamente buscaría revertir esta situación. No necesariamente habría una única manera de hacerlo, y posiblemente diferentes formas presenten beneficios y contras distintos. En un extremo, estaría la posibilidad de elaborar normativas que generen la obligación en los gobernantes de enviar a sus hijos a escuelas públicas. Esta medida estaría muy cerca de garantizar la alineación de incentivos buscada, pero al costo de limitar la libertad de elección de los gobernantes, una decisión que podría ser vista negativamente por aquellos con una mentalidad más libertaria. Una opción más suave podría implicar una simple recomendación hacia los gobernantes y luego hacer pública la elección de cada funcionario (como se hacen públicas las declaraciones de ingresos y capital). La probabilidad de éxito de esta última opción parecería más incierta, pero se dejaría intacta la capacidad de elección de los gobernantes, que solo sufrirían cierta presión sobre su reputación. Una tercera opción que puede implementarse dependiendo del contexto es hacer uso de la norma social descriptiva, es decir, del comportamiento más común. En situaciones en las que la mayoría de los gobernantes manda a sus hijos a escuelas públicas, se pueden enviar cartas 
a los gobernantes que no lo hacen, resaltando el hecho de que son pocos los que se comportan como ellos y que sería deseable que se comprometieran con el desarrollo de las instituciones de educación pública enviando a sus hijos a las escuelas correspondientes. Este tipo de estrategia basada en brindar información sobre la norma social descriptiva encuentra su sustento en hallazgos experimentales de diversos ámbitos, vinculados, por ejemplo, al cuidado de los espacios públicos (Cialdini, Reno y Kallgren, 1990) y al ofrecimiento y la aceptación de coimas (Abbink, Freidin, Gangadharan y Moro, 2016), entre otros, y ha tenido aplicaciones exitosas en campos como la recaudación impositiva (Sousa Lourenço et al., 2016). Veremos más sobre el uso de información de la norma social descriptiva en el apartado siguiente.

\section{EL ENFOQUE NUDGE COMO VIA PARA FACILITAR CONDUCTAS SOCIALMENTE DESEABLES EN LOS GOBERNANTES}

El enfoque Nudge se deriva de los hallazgos de la psicología cognitiva iniciados con la obra de Amos Tversky y continuados por el premio Nobel Daniel Kahneman (Tversky y Kahneman, 1975, 1981, Kahneman 2003, Kahneman, 2011). Estos autores - y muchos otros psicólogos y economistas que siguieron su senda y que fundaron la corriente Behavioural Law and Economics- demostraron que el supuesto de racionalidad completa que suele utilizarse en la economía neoclásica y otras ciencias sociales no se verifica en una gran variedad de situaciones de elección. Las personas no solo contamos con tiempo y capacidad limitada para procesar información, sino que nuestra manera de pensar y tomar decisiones es afectada por "sesgos" sistemáticos que pueden desviar nuestra conducta de las elecciones consideradas "racionales” bajo ciertos estándares (Kahneman, 2011). Así, el exceso de confianza, el anclaje, el sesgo de extrapolación, el error fundamental de atribución, la ilusión de control, la aversión a las pérdidas, el sesgo del statu quo, el lamento de la oportunidad perdida, entre otros, son resortes mentales o actitudes consolidadas frente al riesgo y la incertidumbre que comúnmente exhiben las personas. Los supuestos clásicos de transitividad de las preferencias, cálculo racional de probabilidades y cálculo de la utilidad esperada resultan por tanto empíricamente débiles (Kahneman, 2011). Es más, el enfoque de heurísticas, a la manera de reglas simples y de implementación rápida (Gigerenzer et al., 1999), se ha extendido a la descripción de la toma de decisiones sociales e interactivas, por ejemplo, respecto de la toma de decisiones en situaciones de cooperación (e. g., sostenimiento de bienes comunes; Rand et al., 2014) y también de la toma de decisiones con contenido moral (Baron, 1994; Sunstein, 2005; Young, Willer y Keltner, 2013). 
Basados en la idea de que las personas pueden tomar decisiones guiadas por claves simples del ambiente, Richard Thaler y Cass Sunstein (2008) acuñaron una nueva forma de utilizar los sesgos y heurísticos para modular las elecciones de las personas en direcciones benevolentes y prosociales que denominaron Nudge. Este enfoque consiste en diseñar las opciones de decisión y controlar la información disponible en el ambiente de tal manera que la opción correcta, deseable o prosocial sea escogida con más probabilidad sin necesidad de aplicar sanciones ni prometer recompensas. Un ejemplo ya paradigmático trata sobre la utilización del sesgo del statu quo para incrementar las personas enlistadas para donar órganos. Como suele ocurrir en el enfoque Nudge, la implementación fue muy sencilla: en algunos países se cambió la opción por defecto de una situación en la que las personas debían marcar el recuadro para donar a una opción en que las personas debían marcar el recuadro para no donar. Este cambio fue efectivo y logró incrementar drásticamente las personas que se mostraron dispuestas a donar (Thaler y Sunstein, 2008).

En primera instancia, el enfoque Nudge trata de decisiones individuales que promueven la salud, la riqueza (personal) y el bienestar (Thaler y Sunstein, 2008). El interés en esta perspectiva ha sido tal que gobiernos como el de Barak Obama en los EE. UU. (Obama, 2015) o el de David Cameron en el Reino Unido (The Behavioral Insights Team, 2016) orquestaron equipos de expertos gubernamentales en BI para implementar políticas públicas basadas en evidencia (en el caso de EE. UU., el propio Sunstein presidió la oficina de regulación de servicios públicos durante cuatro años). Si bien el enfoque Nudge se ha utilizado para direccionar de manera libertaria la conducta de los ciudadanos, su utilidad puede extenderse hacia el moldeamiento de la conducta de políticos y gobernantes en sentidos socialmente deseables. A continuación, brevemente presentamos dos ejemplos concretos de implementaciones que podrían ir en este último sentido.

\section{1. Nudge a partir de brindar información adecuada}

La utilización de información sobre normas sociales es una de las modalidades BI más utilizadas por los gobiernos de varios países europeos para lograr comportamientos deseables. Más específicamente, estos países han recurrido a la influencia que tiene la referencia a comportamientos socialmente deseables (normas prescriptivas) o comportamientos mayoritarios (normas descriptivas) sobre las decisiones de las personas en diferentes ámbitos, ya sea conductas de reciclado, uso responsable de fuentes de energía no renovables, hábitos alimentarios saludables o pago de impuestos, entre otros. En este sentido, se busca estructurar 
los ambientes de decisión o brindar elementos informativos que resalten las conductas deseables.

La noción de presentar información sobre la norma social (sea descriptiva o prescriptiva) puede rastrearse en los estudios de campo de Cialdini y colaboradores en los que observaron decrementos sobre conductas como arrojar basura en lugares público (Cialdini et al., 1990) e incluso sobre hurto en parque nacionales (Cialdini et al., 2006). La idea es que el enfocar la atención de las personas sobre un determinado contenido normativo hace más probable que se comporten de manera consistente.

Este tipo de abordaje ha probado ser exitoso en varios estudios de campo y en políticas públicas implementadas (Sousa Lourenço et al., 2016). Un ejemplo de la utilización de información sobre la norma descriptiva (el comportamiento mayoritario) fue implementado con éxito para incrementar la recaudación impositiva en el Reino Unido adjuntando el siguiente mensaje en cartas a los morosos: "Nueve de cada diez personas en Reino Unido pagan sus impuestos a tiempo. Usted está actualmente en la pequeña minoría de personas que no han pagado aún”. Este simple mensaje condujo a un aumento del 5,1 \% en la recaudación en los 23 días de prueba equivalente a 2367 millones de libras (Sousa Lourenço et al., 2016).

Nuestra propuesta es justamente que estas mismas nociones podrían utilizarse para moldear la conducta de los funcionarios públicos en sentidos que sean beneficiosos para la ciudadanía. Más específicamente, la utilización de información normativa parecería ser útil para desalentar la corrupción. Ya existe evidencia experimental de laboratorio que confirma que las personas son susceptibles a cuán común es un determinado comportamiento que daña a un tercero, como es el caso del envió y aceptación de coimas (Kobis et al., 2015; Abbink et al., 2016). En la medida en que la información provista resalta que el comportamiento corrupto es poco común, las personas se ven desalentadas a ganar dinero de maneras que producen externalidades negativas. Estudios experimentales realizados en el Instituto de Investigaciones Económicas y Sociales del Sur (IIESS CONICET Bahía Blanca; Hasrun, Senci, Moro y Freidin, 2017 IIESS working paper) muestran que las personas pueden ser desalentadas a involucrarse en intercambios de coimas cuando se resalta, en el contexto de decisión, información acerca del contenido de la norma prescriptiva (derechos y obligaciones) o información acerca de la función de la norma (evitar externalidades negativas).

Por otra parte, utilizar la información disponible en los ambientes de toma de decisiones podría servir para poner "incentivos” anticorrupción a los políticos. 
En este sentido, algunos autores han sugerido la estrategia de "informar" mejor a los votantes en el momento electoral (Muñoz, 2016). De acuerdo con este enfoque, sería necesario crear una fuente de información neutral, ágil y veraz sobre la corrupción, lo cual permitiría que los electores la pudiesen considerar a la hora de decidir su voto, aumentando de ese modo el valor del precio electoral de la corrupción. Por ejemplo, ello podría hacerse del siguiente modo: aquellos candidatos (que buscan la reelección) que no hubieren enfrentado ninguna acusación de corrupción en la anterior gestión, recibirían en la papeleta una señal verde, los que estuvieran imputados en actos de corrupción recibirían una señal amarilla y los que hubiesen recibido alguna condena que no los inhabilite para el cargo recibirían la señal roja. La clave, por supuesto, es qué agencia determina la colocación de esas señales en la papeleta, ya que el riesgo de que esa información termine siendo manipulada en favor de ciertos intereses partidistas o sectoriales puede llegar a ser más alto que el aumento marginal del precio electoral que hipotéticamente podría traer aparejado el correcto uso de la misma. Pero si los criterios son claros y no sujetos a interpretación, una agencia independiente (formada por miembros de la sociedad civil) podría tal vez hacer alguna diferencia: con solo manejar las señales informativas tal vez se podría aumentar el precio electoral de la corrupción y el valor social de la función pública.

\section{III.2. Nudge a partir de mecanismos de generación de compromiso}

Una de las maneras en que el enfoque Nudge podría utilizarse para desalentar la corrupción en gobernantes y funcionarios públicos en general es a través de mecanismos simples que generen compromiso. En la literatura sobre desempeño laboral, es sabido que los "compromisos públicos" sobre cumplimiento de metas en las empresas, en la forma de mensajes o declaraciones en público, pueden ser efectivos para mejorar la productividad y la eficiencia de los trabajadores. En la misma línea, otros experimentos muestran que las personas que adquieren — por requerimiento - un compromiso público formal, bajan de peso con mayor eficacia que aquellas personas a las que se les paga por hacerlo (Nyer y Dellande, 2009; De Bar et. al, 2011). Un último ejemplo experimental muestra que las personas que firman (es decir, se comprometen) antes de rellenar un autorreporte (y no al final, como es costumbre) exhiben una conducta ética más consistente y honesta que aquellos que rellenan el autorreporte y firman al final (Shu et. al. 2012). A la luz de esta literatura, consideramos que el dispositivo del compromiso público (public commitment) podría extenderse perfectamente al ámbito de la burocracia estatal, y sus efectos deberían ser estudiados con sumo detalle. La forma en que se emiten esos compromisos puede variar: está el formato escrito (en declaraciones juradas) 
o el formato oral, que puede ser emitido en acto público. En principio, cabe esperar que los compromisos en público desplieguen un tipo de entramado informativo (conocimiento común: todos saben, todos saben que saben, etc.) más eficaz que la mera consignación de los compromisos por escrito u online, puesto que en estos últimos el entramado es fragmentado (todos saben, pero no saben que los demás saben también). Por otra parte, cabe esperar que los mecanismos de auditoría social (audiencias públicas con grupos de la sociedad civil prefijados) funcionen de manera más eficaz que los mecanismos burocráticos internos, dadas las posibles implicancias diferenciales sobre la reputación de los involucrados.

En otro orden, sería interesante estudiar el papel que podrían tener los compromisos de gestión. Los compromisos de gestión son “metas” precisas y mensurables que las agencias se plantean al inicio de su mandato y que se comprometen a conseguir antes de finalizar. Las modalidades de las metas, el vínculo causal de la meta con la tarea a cumplir (mediato o inmediato), la forma de los compromisos, su difusión, la forma y mecanismo de control, todo ello puede variar considerablemente dependiendo del formato. En consecuencia, las ciencias del comportamiento encuentran allí un campo riquísimo en el que pueden hacer aportes relevantes.

\section{CONCLUSIONES}

El abordaje de Behavioral Insights está tomando fuerza en gobiernos líderes a nivel mundial (como en la Unión Europea, en el Reino Unido y en EE. UU.), ya que contribuye al programa general de políticas basadas en evidencia y, en particular, permite tener supuestos más realistas acerca de las personas sobre las que las regulaciones se espera sean efectivas.

En este trabajo, presentamos un panorama general del enfoque BI y sus ventajas. Sin duda, el tema presenta entramados y discusiones que no tratamos, como, por ejemplo, qué requisitos son necesarios y suficientes para que la evidencia sea considerada suficientemente buena como para basar una política en ella. Nutley, Powell y Davies (2013) discuten un arreglo jerárquico en cuya cima se encuentra la evidencia provista por revisiones sistemáticas y metaanálisis, ensayos aleatorizados y controlados, bajando a estudios observacionales no aleatorizados y estudio de serie de casos sin controles. Otro tema se da en torno a la generalizabilidad de la evidencia, entre distintos contextos en una misma sociedad e, incluso, entre contextos similares en distintos países. Sin duda, este tipo de discusiones deben ser moduladas por el problema particular que se aborde, las posibles solu- 
ciones que estén bajo consideración, qué se busca conocer, con qué propósito y en qué contexto (Nutley, Powell y Davies, 2013).

En particular, propusimos considerar dos nociones que han recibido apoyo empírico en las ciencias del comportamiento y que podrían ser aplicadas a la regulación de la toma de decisiones de gobernantes y funcionarios públicos en general. Estas nociones tienen que ver con buscar alinear los incentivos de los gobernantes con los de los gobernados para generar bases sólidas sobre las que esperar decisiones que favorezcan a los gobernados, y utilizar el enfoque Nudge para estructurar los ambientes de decisión pública de manera de, por ejemplo, incrementar el compromiso público y minimizar la corrupción.

Con respecto a la idea de alinear los incentivos de los gobernantes con los de los gobernados queremos hacer un comentario a tener en cuenta. De acuerdo con un enfoque estándar, este cometido podría basarse en la utilización de incentivos extrínsecos, es decir, sanciones y recompensas ante comportamientos indeseados y deseables, respectivamente. No obstante, hoy tenemos una comprensión más sofisticada de cómo los incentivos extrínsecos se interrelacionan con las motivaciones intrínsecas, y sabemos que se trata de una relación que puede volverse compleja (Bowles y Polania-Reyes, 2012).

El caso es que cuando se trata de promover la conducta prosocial, no siempre los incentivos extrínsecos logran un incremento de la conducta deseada. Esto se debe a que puede ocurrir que una conducta se encuentre motivada por otros factores. A veces se ha denominado a esto de manera genérica como motivación intrínseca, aunque esta terminología no siempre resulta esclarecedora, ya que a veces la conducta responde a incentivos extrínsecos, simplemente que no son de índole pecuniaria (e.g., consecuencias sobre otros o consecuencias en términos de reputación). No solo se trata de que puede haber diversidad de incentivos detrás del sostenimiento de una conducta prosocial, sino que los incentivos extrínsecos pueden reducir el efecto de las motivaciones intrínsecas (Benabou y Tirole, 2006). Una instancia en que ocurre esto es cuando las personas pasan de centrarse en la motivación intrínseca a centrarse en los incentivos extrínsecos como explicaciones de su propio comportamiento. De esta manera, cuando los incentivos extrínsecos sobre los que se enfocó la persona desaparecen, es común que la conducta prosocial disminuya (Bowles y Polania-Reyes, 2012). No solo eso, sino que en algunos contextos las recompensas monetarias pueden cambiar la manera en que la imagen pública de la conducta es percibida por otros agentes. Si se ofrecen recompensas o se introducen sanciones monetarias para realizar una conducta prosocial sobre la que se tenía alta estima social, la introducción de esos incentivos 
puede dar la señal de que el valor público de esa conducta ya no es tan elevado. En esa línea, es más que elocuente el experimento de Gneezy y Rustichini (2000): en una guardería se empezó a sancionar con una pequeña multa a los padres que llegaban tarde a retirar a sus hijos. Esto, en vez de resultar en un incremento de la puntualidad, resultó en un incremento del número de padres que llegaban tarde y un incremento de la impuntualidad. Una interpretación de este resultado apunta a las señales informativas que proveen los incentivos extrínsecos: los padres no sabían cuán importante era llegar a tiempo a buscar a sus hijos, y asumían (tal vez por ignorancia pluralista ${ }^{5}$ ) que el valor de la imagen pública era alto. Cuando se introdujeron las multas, se percataron de que el valor de ser puntual tenía un precio reducido y las personas actualizaron sus creencias sobre ese valor, derivando en una conducta contraria a la buscada. En breve, la manera de buscar alineación de incentivos entre gobernantes y gobernados requiere que estemos atentos a los conocimientos sobre la interrelación entre distintos tipos de incentivos y sus efectos sociales.

Nuestra propuesta en este artículo es de índole general y busca resaltar algunos instrumentos conceptuales que han mostrado ser efectivos en modular la conducta de las personas, y, por ende, podrían ser utilizados en pos de conducir las decisiones de los gobernantes en sentidos socialmente deseables. En este enfoque general, sin embargo, no analizamos problemas específicos que pueden surgir al intentar introducir conocimiento científico en la agenda política y gubernamental (a los interesados en estos temas les recomendamos leer Carney, 2016).

En síntesis, hemos resaltado las ventajas de utilizar las ciencias del comportamiento a servicio de la generación de políticas basadas en evidencia, y en particular marcamos dos temáticas que pueden ser relevantes a la hora de elaborar políticas efectivas. En este contexto, sugerimos que estas herramientas también podrían utilizarse para conducir y enmarcar la toma de decisiones de los gobernantes en sentidos sociales deseables. Esperamos ver más líneas de investigación en esta dirección en el futuro cercano, y que los gobiernos locales, provinciales y nacionales en la Argentina y en Sudamérica comiencen a obtener ventajas de cooperar con científicos que trabajan en las ciencias de la conducta.

5 La ignorancia pluralista es una situación en la que la mayoría de un grupo rechaza en privado una norma de conducta grupal pero incorrectamente asume que los demás la aceptan, y por lo tanto todos prefieren seguir realizando la conducta pese a que una mayoría la rechazaría si conociera lo que los demás piensan (Katz y Allport, 1931; Prentice y Miller, 1993). 


\section{REFERENCIAS BIBLIOGRÁFICAS}

Abbink, K., Freidin, E., Gangadharan, L., \& Moro, R. (2016). The effect of social norms on bribe offers. IIESS CONICET Bahía Blanca, Working Paper $\mathrm{N}^{\circ}$ 2. Recuperado de http://www.iiess-conicet.gob.ar/index.php/publicaciones-grales/documentos-de-trabajo

Baron, J. (1994). Nonconsequentialist decisions. Behavioral and Brain Sciences, $17,1-42$.

Benabou, R., \& Tirole, J. (2006). Incentives and Prosocial Behavior. American Economic Review, 96 (5), 1652-1678.

Bowles, S., \& Polanía-Reyes, S. (2012). Economic incentives and social preferences: substitutes or complements? Journal of Economic Literature, 50 (2), 368-425.

Carney, P. (2016). The Politics of Evidence-Based Policy Making. London: Palgrave Macmillan.

Cialdini, R. B., Reno, R. R., \& Kallgren, C. A. (1990). A focus theory of normative conduct: Recycling the concept of norms to reduce littering in public places. Journal of Personality and Social Psychology, 58, 1015-1026. doi:10.1037/0022-514.58.6.1015.

Cialdini, R. B., Demaine, L. J., Sagarin, B. J., Barrett, D., W., Rhoads, K., \& Winter, P. L. (2006). Managing social norms for persuasive impact. Social Influence, 1 (1), 3-15.

Costas-Pérez, E., Solé-Ollé, A., \& Sorribas-Navarro, P. (2012). Corruption scandals, voter information, and accountability, European Journal of Political Economy, 28 (4), 469-484.

DeBar, L. L., Schneider, M., Drews, K., Ford, E. G., Stadler, D.D., Moe, E. L., et al. (2011). Student public commitment in a school-based diabetes prevention project: impact on physical health and health behavior. $B M C$ Public Health, 2-11. Recuperado de http://www.biomedcentral.com/14712458/11/711

Gigerenzer, G., Todd, P. M., \& the ABC Research Group (1999). Simple Heuristics That Make Us Smart. New York: Oxford University Press.

Gneezy, U., \& Rustichini, A. (2000). A Fine Is a Price. Journal of Legal Studies, 29 (1), 1-18.

Hasrun, H., Senci, C. M., Moro, R., \& Freidin, E. (2017). The effect of prescriptive norms and negative externalities on bribery decisions. IIESS CONICET Bahía Blanca, Working Paper N. ${ }^{\circ}$ 3. Recuperado de http://www.iiess-conicet.gob.ar/index.php/publicaciones-grales/documentos-de-trabajo

Kahneman, D. (2003). A psychological perspective on economics. The American Economic Review, 93 (2), 162-168. 
Kahneman, D. (2011). Pensar rápido, pensar despacio. Barcelona: Debate. Katz, D., \& Allport, F H. (1931). Student Attitudes. Syracuse, New York.: Craftsman. Kobis, N. C., van Prooijen, J. W., Righetti, F., \& Van Lange, P. A. M. (2015). Who doesn't? - The impact of descriptive norms on corruption. PLOS ONE, 10 (6), e0131830. doi: 10.1371/journal.pone.0131830

López Guerra, C. (2016). Piloting Responsibility and Intergenerational Justice. En I. González Ricoy \& A. Gosseries (Eds.). Institutions for Future Generations (299-311). New York: Oxford University Press,

Muñoz, J. (2013). El precio electoral de la corrupción: ¿Por qué los votantes castigan tan poco? Pasajes, 42. Recuperado de http://www.revistasculturales. com/xrevistas/PDF/24/1709.pdf

Nyer, P., \& Dellande, S. (2010). Public commitment as a motivator to weigh loss. Psychology and Marketing, 27 (1), 1-10.

Nutley, S., Powell, A., \& Davies, H. (2013). What counts as good evidence? London: Alliance for Useful Evidence. Recuperado de http://www.alliance4usefulevidence.org/assets/What-Counts-as-Good-Evidence-WEB.pdf.

Obama, B. (2015). Executive Order - Using Behavioral Science Insights to Better Serve the American People. Recuperado de https://www.whitehouse.gov/ the-press-office/2015/09/15/executive-order-using-behavioral-science-insights-better-serve-american

Prentice, D. A., \& Miller, D. T. (1993). Pluralistic ignorance and alcohol use on campus: Some consequences of misperceiving the social norm. Journal of Personality and Social Psychology. 64 (2), 243-256.

Rand, D. G., Peysakhovich, A., Kraft-Todd, G., Newman, G. E., Wurzbacher, O., Nowak, M. A., \& Greene, J. (2014). Social heuristics shape intuitive cooperation. Nature Communications, 5 (3677). doi: 10.1038/ncomms4677

Rivero Rodríguez, G. \& Fernández Vázquez, P. (2011). Las consecuencias electorales de los escándalos de corrupción municipal, 2003-2007. Estudios de Progreso N.o 59. Madrid: Fundación Alternativas. Recuperado de: http:// www.fundacionalternativas.org/public/storage/estudios_documentos_archivos/a4112d386ee4ebfa92dae911711f0de3.pdf

Shu, L., Mazar, N., Gino, F., Ariely, D., \& Bazerman, M. (2012). Signing at the beginning makes ethics salient and decreases dishonest self-reports in comparison to signing at the end. Proceedings of the National Academy of Sciences, 109 (38), 15197-15200.

Sousa Lourenço, J., Ciriolo, E., Rafael Almeida, S., \& Troussard, X. (2016). Behavioural insights applied to policy: European Report 2016. Joint Research Centre, EUR 27726 EN. doi: 10.2760/903938.

Sunstein, C.R. (2005). Moral heuristics. Behavioral and Brain Sciences, 28 (4), 531-573. 
Thaler, R. H. \& Sunstein, C. R. (2008). Nudge: Improving decisions about health, wealth, and happiness. New Haven \& London: Yale University Press.

The Behavioral Insights Team (2016). Update Report 2015-16. Recuperado de: http://www.behaviouralinsights.co.uk/

Tversky, A., \& Kahneman, D. (1975). Judgment under uncertainty: Heuristics and biases. En D. Wendt y C. Vleck (Eds.), Utility, probability, and human decision making. Theory and Decision Library, (pp. 141-162). Netherlands: Springer.

Tversky, A., \& Kahneman, D. (1981). The Framing of Decisions and the Psychology of Choice. Science, 211 (4481), 453-458.

Young, O. A., Willer, R., \& Keltner, D. (2013). Thou Shalt Not Kill: Religious Fundamentalism, Conservatism, and Rule-Based Moral Processing. Psychology of Religion and Spirituality, 5 (2), 110-115.

(C) 2017 por los autores; licencia otorgada a la revista Estudios económicos. Este artículo es de acceso abierto y distribuido bajo los términos y condiciones de una licencia Atribución-No Comercial 3.0 Unported (CC BY-NC 3.0) de Creative Commons. Para ver una copia de esta licencia, visite http://creativecommons.org/ licenses/by-nc/3.0/ 